\title{
Bilosomes as Promising Nanovesicular Carriers for Improved Transdermal Delivery: Construction, in vitro Optimization, ex vivo Permeation and in vivo Evaluation
}

This article was published in the following Dove Press journal:

International Journal of Nanomedicine

\section{Sadek Ahmed (iD \\ Mohamed Aly Kassem ${ }^{\dagger}$ \\ Sinar Sayed (D)}

Department of Pharmaceutics and Industrial Pharmacy, Faculty of Pharmacy, Cairo University, Cairo, Egypt

†Mohamed Aly Kassem, passed away on August 21, 2020

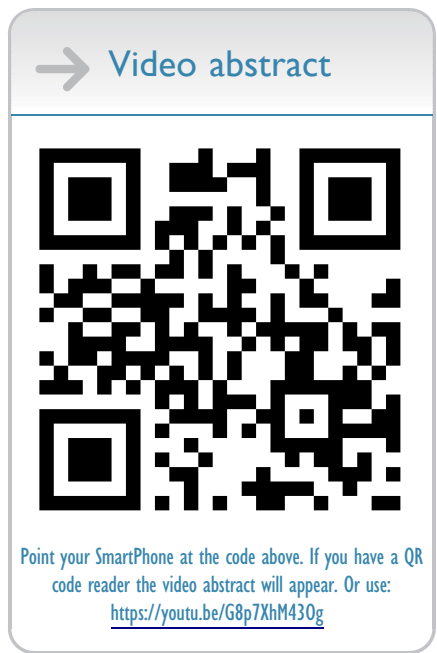

Correspondence: Sinar Sayed Faculty of Pharmacy, Cairo University, Kasr El-Aini, Cairo I I562, Egypt $\mathrm{Tel}+201010421543$

Email sinar.fouad@pharma.cu.edu.eg
Purpose: The goal of this research was to enhance the transdermal delivery of lornoxicam (LX), using nanovesicular carriers composed of the bile salt sodium deoxycholate (SDC), soybean phosphatidyl choline (SPC) and a permeation enhancer limonene.

Methods: Thin-film hydration was the technique employed for the fabrication using a BoxBehnken design with three central points. The investigated factors were SPC molar concentration, SDC amount in $\mathrm{mg}$ and limonene percentage (\%). The studied responses were percent entrapment efficiency (\%EE), particle size (PS), polydispersity index (PDI), zeta potential (ZP), and in vitro drug release (after 2, $10 \mathrm{~h}$ ). In order to obtain the optimum formula, numerical optimization by Design-Expert ${ }^{\circledR}$ software was used. Electing the optimized bilosomal formula was based on boosting \%EE, ZP (as absolute value) and in vitro drug release, taking in consideration diminishing PS and PDI. Further assessment of the selected formula was achieved by transmission electron microscopy (TEM), differential scanning calorimetry (DSC), Fourier transform infrared spectroscopy (FTIR), stability testing, ex vivo skin permeation and deposition. The in vivo pharmacodynamics activities of the optimized formula were examined on male rats and mice and compared to that of the oral market product.

Results: The optimized bilosomal formula demonstrated to be nonirritant, with noticeably enhanced anti-inflammatory and antinociceptive activities. Superior in vivo permeation was proved by confocal laser scanning microscopy (CLSM).

Conclusion: The outcomes demonstrated that bilosomes could improve transdermal delivery of lornoxicam.

Keywords: lornoxicam, permeation enhancer, factorial design, confocal laser scanning microscopy, antinociceptive

\section{Introduction}

Recent techniques in drug development programs have guided the introduction of many molecules with insignificant aqueous solubility and inadequate bioavailability profile into the market. ${ }^{1}$ Low aqueous solubility not only yields irreproducible therapeutic effect, but also wastes large amounts of drugs. ${ }^{2}$ It was stated that $70 \%$ of the new drug candidates were discarded in early stages due to poor solubility in water before they ever reached the pharmaceutic department. ${ }^{3}$ Poor solubility also causes bioavailability problems due to the unexpected sedimentation of drugs in 
aqueous biological fluid. Nanotechnology provides many benefits to old-style drug design, delivery and medical diagnostics. There has been recent achievement in the use of nanoparticles as platforms or carriers for poorly soluble drugs.

Lornoxicam is an oxicam derivative and belongs to nonsteroidal anti-inflammatory drugs (NSAIDs). ${ }^{4}$ Its shows powerful analgesic and antipyretic effects owing to its nonselective inhibition of COX-1 and COX-2., ${ }^{5,6}$ It is used in rheumatoid arthritis, osteoarthritis, sciatica, dental pain and postoperative pain management. ${ }^{7,8}$ It has insignificant aqueous solubility and rapid clearance $\left(\mathrm{t}_{0.5}=3-4 \mathrm{~h}\right)$. It has potential gastrointestinal, liver, cardiac and renal toxicities. ${ }^{9}$ Many approaches were previously used to overcome the side effects of LX such as liposomes, ${ }^{9}$ proniosomal gel, ${ }^{10}$ and niosomes. ${ }^{11}$

Transdermal delivery system provides an approach to avoid the side effects of NSAIDs and extend duration of action. Stratum corneum (SC) represents the barrier which protects the skin and prevents the absorption of drugs. However, recent technologies provide several approaches to transverse SC especially through nanosystems such as liposomes, ${ }^{12}$ ethosomes, ${ }^{13}$ transferosomes, ${ }^{14}$ and bilosomes. ${ }^{15}$

Bilosomes (bile salts stabilized vesicles) represent a recent vesicular carrier that was first described by Conacher et al. ${ }^{16}$ They are closed vesicles composed of nonionic surfactant resembling niosomes but containing bile salts. ${ }^{15}$ Bilosomes provide a significant oral delivery of vaccines because they provide better protection against gastrointestinal bile salts and enzymes. ${ }^{17-19}$ Bilosomes have nanosized PS which is required for optimum transdermal delivery. ${ }^{15}$ The use of negative charged bile salts (like sodium deoxycholate) results in increased stability of the formed vesicular system. It also has a fluidizing effect which would enhance transdermal delivery. Limonene is a lipophilic terpene which can improve skin permeation by interacting with stratum corneum lipids. The employment of a bilosomal system for transdermal delivery revealed an enhanced permeability through SC which is a necessary requirement for transdermal delivery. ${ }^{15}$ Moreover, its safety was previously confirmed through histopathological study. ${ }^{15}$

Factorial designs provide an excellent solution for indicating response and interaction of many factors. It also allows numerical optimization which permits discovery of the best formula through preferred condition of each response. $^{20}$

For our knowledge the employment of bilosomes to enhance transdermal delivery of lornoxicam has not been mentioned before. The goal of this study is to formulate a sustained release bilosomal system through a Box-Behnken design followed by numerical optimization and characterization of the selected formula. Ex vivo permeation study of the optimized bilosomal formula was conducted in order to assess permeation parameters of LX from this system. Skin irritancy test and histopathological study was conducted to ensure the safety of the formed bilosomes to human skin. Efficacy was tested through studying the anti-inflammatory effect, antinociceptive activity and in vivo permeation of the optimized bilosomal formula.

\section{Materials and Methods}

\section{Materials}

LX received as a gift from Delta Pharma, 10th of Ramadan City, Cairo, Egypt. Bile salt sodium deoxycholate (SDC), soybean phosphatidyl choline (SPC), limonene, chloroform, methanol, acetonitrile (HPLC grade) and rhodamine B were bought from Sigma Chemical Company. Ethanol (95\%) was provided from El-Nasr Pharmaceutical Chemicals Co. (Cairo, Egypt). All other chemicals and solvents were of analytical grade and were used as received.

\section{Methods}

\section{Construction of LX-loaded Bilosomes}

Bilosomes of LX were constructed using a SDC, SPC and limonene based on thin-film hydration technique ${ }^{15,21}$ with slight modification. Concisely, LX $(8 \mathrm{mg})$, SPC $(0.02$, 0.04 , or $0.06 \mathrm{M})$ and limonene $(0.25,0.5$, or $0.75 \%$ ) were precisely weighed and mixed using $(10 \mathrm{~mL})$ chloroform and methanol combination (2:1) in a one-liter round-bottomed flask. The organic phase was gradually vaporized under vacuum at $60 \pm 0.5^{\circ} \mathrm{C}$ for $(15 \mathrm{~min})$ using a rotary evaporator (Rotavapor, Heidolph VV 2000, Burladingen, Germany) at $120 \mathrm{rpm}$. The formed thin film was then rehydrated with $10 \mathrm{~mL}$ phosphate buffer ( $\mathrm{pH} 7.4)$ containing SDC $(10,20$, or $30 \mathrm{mg})$ at $60 \pm 0.5^{\circ} \mathrm{C}$ for half an hour under atmospheric pressure. Table 1 shows the structure of the constructed bilosomes formulae (T1-T15).

\section{Experimental Design for LX-loaded Bilosomes}

Box-Behnken with three central points for each formula was used for preparing investigated formulae (T1-T15). The independent variables studied were: (A) SPC molar concentration at three levels; (B) SDC amount at three levels; and (C) limonene percentage at three levels. These levels were described as $(-1,0,+1)$ and their matching concrete values are given in Table 2. Selection 


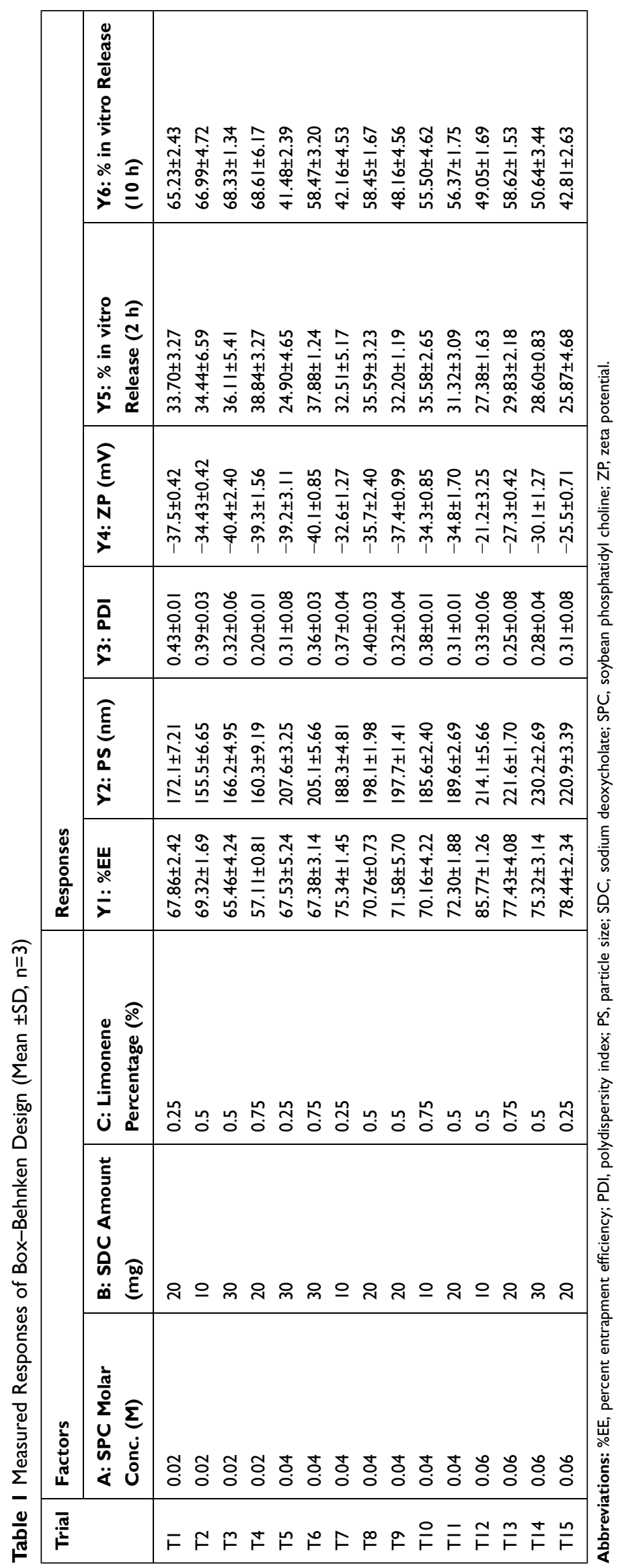


Table 2 Box-Behnken Design Used for Optimization of Bilosomal Formulae

\begin{tabular}{|l|l|l|l|}
\hline \multirow{2}{*}{ Factor (Independent Variable) } & \multicolumn{2}{l|}{ Level } \\
\cline { 2 - 4 } & $-\mathbf{I}$ & $\mathbf{0}$ & $\mathbf{+ I}$ \\
\hline A: SPC molar concentration (M) & 0.02 & 0.04 & 0.06 \\
B: SDC amount (mg) & 10 & 20 & 30 \\
C: limonene percentage (\%) & 0.25 & 0.5 & 0.75 \\
\hline Response (Dependent Variable) & Desirability Constraints \\
\hline YI: \%EE & Maximize \\
Y2: PS (nm) & Minimize \\
Y3: PDI & Minimize \\
Y4: ZP (absolute value) $(\mathrm{mV})$ & Maximize \\
Y5: \% in vitro release $(2 \mathrm{~h})$ & Maximize \\
Y6: \% in vitro release $(10 \mathrm{~h})$ & Maximize \\
\hline
\end{tabular}

Abbreviations: \%EE, percent entrapment efficiency; PDI, polydispersity index; PS, particle size; SDC, sodium deoxycholate; SPC, soybean phosphatidyl choline; ZP, zeta potential.

of these levels was based on preliminary trials. But additional variables such as: amount of drug $(8 \mathrm{mg})$, hydration volume $(10 \mathrm{~mL})$, temperature of both hydration and film preparation were kept untouched. The investigated dependent variables were: percent entrapment efficiency (\%EE), particle size (PS), polydispersity index (PDI), zeta potential (ZP), and in vitro release (after 2, $10 \mathrm{~h}$ ) (Y1, Y2, Y3, Y4, Y5, Y6, respectively). ANOVA to realize the significance of each factor was done by Design-Expert ${ }^{\circledR}$ software version 7 (Stat-Ease, Inc., Minneapolis, MN, USA).

\section{In vitro Characterization of LX-loaded Bilosomes Percent Entrapment Efficiency (\%EE)}

The $\% \mathrm{EE}$ of LX was calculated indirectly by measuring the free LX (nonencapsulated LX) in the dispersion medium. One milliliter of dispersion medium was subjected to centrifugation via a cooling centrifuge (3K30, Sigma, Germany) at 15,000 rpm for one hour at $4{ }^{\circ} \mathrm{C}$. The supernatant was detached, diluted with ethanol (95\%). The concentration of free LX was spectrophotometrically calculated (Shimadzu, model UV-1601 PC, Kyoto, Japan) at $\lambda \max 381 \mathrm{~nm}$ via the calibration curve $\left(n=3, R^{2}=0.9999\right)$. Each formula was tested three times. The $\% \mathrm{EE}$ was computed using the subsequent formula: ${ }^{21-23}$

$$
\% \mathrm{EE}=\frac{(\text { total amount of } \mathrm{LX}-\text { total amount of free LX) }}{\text { total amount of LX }}
$$

Total amount of LX is the actual weighed amount ( $8 \mathrm{mg}$ ), total amount of free LX (amount of LX in supernatant)
Particle Size (PS), Polydispersity Index (PDI) and Zeta Potential (ZP)

At the beginning the dispersions were subjected to suitable dilution by deionized water. ${ }^{24}$ Then, the mean PS, ZP, and PDI of the constructed formulae were obtained by Zetasizer Nano ZS (Malvern Instruments, Malvern, UK) using the dynamic light-scattering technique at $\left(25 \pm 2^{\circ} \mathrm{C}\right)$ employing a helium-neon laser with a wavelength of 633 $\mathrm{nm}$. Each formula was assessed in triplicate.

\section{In vitro Release Studies}

Bag diffusion technique was adopted for determination of LX in vitro release. ${ }^{23,25}$ Briefly, a dialysis bag (immersed overnight in release medium) containing $2 \mathrm{~mL}$ (equivalent to $1.6 \mathrm{mg} \mathrm{LX}$ ) of each formula or LX suspension was then positioned in $50 \mathrm{~mL}$ release medium (phosphate buffer $\mathrm{pH}=6.8)$ in amber bottles. Bottles are then positioned in shaker functioning at fixed temperature of $\left(37 \pm 0.5^{\circ} \mathrm{C}\right)$ and at $100 \mathrm{rpm}^{23,26}$ Samples of $3 \mathrm{~mL}$ were removed from the medium at interval of $(0.5,1,2,4,6,8,10$, and $24 \mathrm{~h})$ and substituted with equivalent volume of newly formed release medium to preserve sink state. Percentage of drug released was calculated and charted against time. The percentage released was carried out by spectrophotometric measurement (Shimadzu, model UV-1601 PC, Kyoto, Japan) at $\lambda \max 381 \mathrm{~nm}$ using the calibration curve $(\mathrm{n}=3$, $\left.\mathrm{R}^{2}=0.9995\right)$. The drug release profiles were tailored to zero, first, and Higuchi diffusion models. ${ }^{27}$ The largest coefficient $\left(\mathrm{R}^{2}\right)$ suggests the appropriate model. ${ }^{28}$

\section{Choosing the Optimized Bilosomal Formula}

Numerical optimization by Design-Expert ${ }^{\circledR}$ software version 7 (Stat-Ease, Inc., Minneapolis, MN, USA) was used to determine desirability taking into consideration the significant factors and avoiding the nonsignificant ones. Choosing this formula was based on maximizing \%EE, ZP (as absolute value) and in vitro release, in addition to minimizing PS and PDI. The formula with highest desirability (closest to one) was taken for further examination. ${ }^{21}$

\section{Characterization of the Optimized Bilosomal Formula \\ Lyophilization of the Optimized Bilosomal Formula}

Firstly, optimized bilosomal formula was frozen at $\left(-20^{\circ} \mathrm{C}\right)$ followed by lyophilization at $\left(-45^{\circ} \mathrm{C}\right)$ under lowered pressure for $(24 \mathrm{~h})$ (Novalyphe-NL 500 freeze-dryer, Savant Instruments, NY, USA). 


\section{Differential Scanning Calorimetry (DSC)}

Thermal characters of LX, SDC, limonene, SPC and lyophilized optimized bilosomal formula were acquired by heating (3-4 mg) of each sample to $\left(350^{\circ} \mathrm{C}\right)$ in a nitrogen environment in an aluminum pan at a scanning rate of $\left(10^{\circ}\right.$ $\mathrm{C} / \mathrm{min}$ ), Thermograms were recorded by (DSC7, PerkinElmer, Waltham, MA, USA. ${ }^{29}$

\section{Fourier Transform Infrared Spectroscopy (FTIR)}

Bruker FTIR spectrophotometer (Model 22; Bruker, Coventry, UK) was used to assess the possibilities of drug-excipient interaction. The samples of LX, SDC, SPC, and lyophilized optimized bilosomal formula were made by $\mathrm{KBr}$ pellet technique and were studied at transmission mode over wave number from 4000 to $400 \mathrm{~cm}^{-1}$.

\section{Transmission Electron Microscopy (TEM)}

The morphological appearance of the optimized bilosomal formula was studied using TEM (Jeol JEM1230, Tokyo, Japan) after proper dilution. One drop of the optimized dispersion was adsorbed on copper grid, stained with $1 \%$ phosphotungstic acid which provides a negative charge and then dried at $\left(25 \pm 0.5^{\circ} \mathrm{C}\right)$ for $10 \mathrm{~min}$ prior to TEM scanning.

\section{Gel Formation}

An accurate amount of carpabol $(1 \% \mathrm{w} / \mathrm{w})$ was added slowly to the optimized bilosomal formula or LX suspension and stirred continuously using a magnetic stirrer to form a gel. The gel was stored overnight in the refrigerator to obtain a clear gel. ${ }^{30}$

\section{Effect of Storage}

To determine the consequence of storage on optimized bilosomal formula, it was kept in a firmly capped glass vial and stored at $\left(4-8^{\circ} \mathrm{C}\right)$ for three months. ${ }^{31}$ By the end of storage period, it was assessed with respect to its appearance, \%EE, PS, ZP and percent released (2, $10 \mathrm{~h}$ ). All tests were done in triplicate.

\section{HPLC Detection of LX}

Previously stated validated HPLC method was adopted ${ }^{32}$ with slight modification. The device consisted of an Agilent 1260 infinity series connected to a DAD detector with a quaternary pump and an injector. The study was performed using RP-C18 column (250 X 4.6 mm ODS (2), spherisorb $5 \mathrm{~mm}$ ). Mobile phase comprised of (acetonitrile: water) in (70:30) ratio. Mobile phase was previously subjected to sonication, filtration and degassing then run at velocity of $1 \mathrm{~mL} / \mathrm{min}$ at room temperature. LX was identified at $381 \mathrm{~nm}$ and the injection volume was $20 \mu \mathrm{L}$. Validation of method was carried out in terms of linearity, precision, and accuracy with regression coefficient $\left(\mathrm{R}^{2}=0.9996\right)$.

\section{Ex vivo Permeation and Skin Deposition Studies}

The study performed was agreed by Research Ethics Committee (REC), Faculty of Pharmacy, Cairo University (approval number PI 2377), followed Guide for Care and Use of Laboratory Animals published by the US National Institute of Health (NIH Publication No. 85-23, revised 2011). This examination was accomplished by skin from anesthetized recently born albino rats. The residual attaching fat was removed by isopropyl alcohol. Then the skin was rinsed with deionized water, covered with aluminum foil then preserved at $\left(-20^{\circ} \mathrm{C}\right) .^{33}$ The skin was attached to one end of the open-ended tube and a certain amount of donor medium (optimized bilosomal gel or LX suspension gel) containing an equivalent amount of $1.6 \mathrm{mg}$ of LX was added. Phosphate buffer $(\mathrm{pH}=6.8)$ resembled the receptor medium. At planned intervals (1, $2,4,6,8,10 \mathrm{~h})$ samples of $(0.5 \mathrm{~mL})$ were removed and substituted quickly with new receptor medium to maintain sink condition. LX was quantified in the withdrawn samples using the aforementioned HPLC method. A plot of the amounts of LX permeated through the membrane per unit area $\left(\mu \mathrm{g} / \mathrm{cm}^{2}\right)$ vs time $(\mathrm{h})$ was drawn. Cumulative amount of LX permeated through the membrane per unit area after $10 \mathrm{~h}(\mathrm{Q} 10 \mathrm{~h})$, average flux value after $10 \mathrm{~h}\left(\mathrm{~J}_{\max }\right)$, the enhancement ratio (ER) and amount retained in skin after $10 \mathrm{~h}$ were determined for both optimized bilosomal gel and LX suspension gel. All studies were performed in triple. The flux $\left(\mathrm{J}_{\max }\right)$ and the enhancement ratio (ER) were calculated from the following equations. ${ }^{34,35}$

$$
\begin{aligned}
& J_{\max }=\frac{\text { Amount of drug permeated }}{\text { Time X Area }} \\
& E R=\frac{\text { Jmax of formulation }}{\text { Jmax of Plain gel (control) }}
\end{aligned}
$$

Parameters of flux are: cumulative amount of LX permeated, total time of experiment and area of membrane. At the end of the permeation study, Skin samples were washed cautiously with $10 \mathrm{~mL}$ normal saline to get rid of the adhering gel formulation. ${ }^{36}$ After that, samples were cut into pieces and sonicated in $50 \mathrm{~mL}$ phosphate buffer $\mathrm{pH}=6.8$ for $30 \mathrm{~min}$ to leach out the deposited drug. The samples were centrifuged and the supernatant was filtered 
through a $0.45 \mu \mathrm{m}$ membrane filter and measured by HPLC.

\section{In vivo Evaluation}

Evaluation of Anti-inflammatory Activity

This test was done according to Ammar et al. ${ }^{37}$ Four groups of male Wistar rats $(200 \pm 50 \mathrm{~g})$ each containing six rats were used. No medication was given to group I (negative control group). Market oral LX tablets (Lornox ${ }^{\circledR}$ tablet) was given to group II. Third and fourth groups received placebo and optimized bilosomal gel respectively after shaving their backs. Observation of all groups was done at the same time. Four percent formaldehyde solution was used to induce localized inflammation through sub-plantar injection of $0.1 \mathrm{~mL}$ into the left footpad of the rat's hind paw, $30 \mathrm{~min}$ before drug administration to ensure maximum edema was acquired. ${ }^{38}$ Edema size was measured by plethysmometer at $(0,1,2,4,6,24,48$, and $72 \mathrm{~h}) .{ }^{39}$

\section{Evaluation of Antinociceptive Activity}

Analgesic action was examined through abdominal constriction induced by acetic acid. ${ }^{37}$ However, this technique was subjected to some alteration stated by Adzu et al. ${ }^{40}$ Mice ( 25 $\pm 5 \mathrm{~g}$ ) were divided into three groups (four mice per group). The first group received no treatment (negative control). Market product (Lornox ${ }^{\circledR}$ tablet) was received by the second group. The third group received optimized bilosomal gel after shaving their back. Each mouse was injected with a single dose of $10 \mathrm{~mL} / \mathrm{kg}$ of $0.7 \%$ aqueous solution of acetic acid via intraperitoneal route $30 \mathrm{~min}$ following the drug application. Each mouse was located in a clear observation cage. Abdominal constriction (stretching of hind limbs) within 5 and 15 min after injection of acetic acid was considered. Activity was indicated as percent reduction of nociception (reduction in attacks of writhing) between three groups. ${ }^{41}$

\section{Skin Irritancy Test}

Potential localized reaction between the optimized bilosomal gel and the skin was examined through skin irritancy test. Briefly, Wistar rats $(200 \pm 50 \mathrm{~g})$ were divided into two groups each containing three rats. The backs of all the rats were shaved. No medication was given to the first group (negative control group). Optimized bilosomal gel was applied on the shaved back of the second group. Both groups were examined visually for any modification, as erythema, for $48 \mathrm{~h}$. Skin irritation of the both groups was observed according to Draize scale as follows: 0 points: no erythema, 1 point: very slight erythema, 2 points: well defined erythema, 3 points: moderate to severe erythema, and 4 points: severe erythema. ${ }^{42}$

\section{Histopathological Study}

Possible ultrastructural modifications and irritation reactions in the skin after application of the optimized bilosomal gel were assessed through histopathological study. Briefly, three groups of male Wistar rats $(200 \pm 50 \mathrm{~g})$ each containing four rats were used. Group I received normal saline (negative control). Group II took optimized bilosomal gel. Group III acted as positive control through application of the irritating agent isopropanol. Application of each medication was continued for one week, three times daily. The skin was then removed for evaluation as mentioned by Bancroft et al. ${ }^{43}$ Briefly, skin was preserved in $10 \%$ formalin for one day then rinsed with water and dehydrated by serial dilutions of alcohol (methyl, ethyl, and absolute ethyl). Later, cleared by xylene then embedded in paraffin beeswax blocks and stored at $56^{\circ} \mathrm{C}$ for $24 \mathrm{~h}$, whereupon 4-mm thick sections were sliced by a microtome (Leica Microsystems SM2400, Cambridge, UK), deparaffinized, stained with $\mathrm{H} \& \mathrm{E}$ and then examined via a light microscope.

\section{In vivo Permeation}

The penetration depth of bilosomes through the mice skin was assessed via confocal laser scanning microscopy (CLSM, LSM 710, Carl Zeiss, Jena, Germany). ${ }^{44,45}$ Rhodamine B $(\mathrm{RhB})$ was loaded in the formula instead of LX at concentration of $(0.1 \mathrm{w} / \mathrm{w} \%)$ then applied as gel on albino mice skin $(0.5 \mathrm{~g})$ for three hours, $0.1 \% \mathrm{w} / \mathrm{w} \mathrm{RhB}$ solution gel was used as a reference. The mice were then sacrificed by cervical dislocation. The skin was carefully removed, washed with $10 \%$ ethanol then lightly wiped. ${ }^{46,47}$ The excised skin was inserted between a glass slide and a cover slip. The sample slides were then investigated using CLSM to estimate the penetrative depth of $\mathrm{RhB}$ bilosomal gel and compared to $\mathrm{RhB}$ solution gel. $\mathrm{RhB}$ fluorescence was excited at $\lambda \max =485 \mathrm{~nm}$ and distinguished at $\lambda \max =595 \mathrm{~nm}^{46}$

\section{Result and Discussion Analysis of Factorial Design}

Factorial designs have the ability to determine the consequence of several factors on the characters of delivery systems. ${ }^{48}$ Each response was tested individually and tailored to various order models. ${ }^{49}$ For all the examined responses, the predicted $\mathrm{R}^{2}$ values were in sensible harmony with the adjusted $\mathrm{R}^{2}$, as shown in Table 3. Adequate precision $(>4)$ 
Table 3 Output Data of Box-Behnken Factorial Analysis

\begin{tabular}{|c|c|c|c|c|c|c|}
\hline Response & $\mathbf{R}^{2}$ & $\begin{array}{l}\text { Adjusted } \\
\mathbf{R}^{2}\end{array}$ & $\begin{array}{l}\text { Predicated } \\
\mathbf{R}^{2}\end{array}$ & $\begin{array}{l}\text { Adequate } \\
\text { Precision }\end{array}$ & $\begin{array}{l}\text { Significant } \\
\text { Factors }\end{array}$ & Final Coded Equation \\
\hline \%EE & 0.9381 & 0.9037 & 0.8218 & 19.060 & $A, B, C, A C, C^{2}$ & $\begin{array}{l}\% E E=72.93+7.15 A-3.1 I B-2.14 C \\
+2.44 A C-2.77 C^{2}\end{array}$ \\
\hline PS (nm) & 0.9702 & 0.9653 & 0.9524 & 38.583 & $A, B$ & $P S=194.19+29.09 A+8.02 B$ \\
\hline $\mathrm{ZP}(\mathrm{mV})$ & 0.9702 & 0.9621 & 0.9446 & 32.488 & $A, B, A^{2}$ & $Z P=36.3-5.91 \mathrm{~A}+3.44 \mathrm{~B}-4.36 \mathrm{~A}^{2}$ \\
\hline $\begin{array}{l}\% \text { drug released }(2 \\
\text { h) }\end{array}$ & 0.8806 & 0.8481 & 0.7556 & 16.373 & $A, C, B C$ & $\begin{array}{l}\% \text { Release }(2 h)=32.32-3.93 A+3.14 C \\
+2.48 B C\end{array}$ \\
\hline $\begin{array}{l}\text { \% drug released } \\
(\mathrm{IO} \mathrm{h})\end{array}$ & 0.8695 & 0.8339 & 0.7637 & $|4.81|$ & $A, C, A^{2}$ & $\begin{array}{l}\% \text { Release }(10 h)=51.53-8.5 I A+6.19 C \\
+7.26 \mathrm{~A}^{2}\end{array}$ \\
\hline
\end{tabular}

Abbreviations: \%EE, percent entrapment efficiency; PS, particle size; ZP, zeta potential.

was obtained in almost all responses navigating that the model is valid to lead the design space. ${ }^{21}$

\section{Effect of Preparation Variables on \%EE}

Encapsulating significant quantity of LX is a meaningful character of bilosomes for its promising use as a transdermal delivery system. The \%EE fluctuated between (57.11 \pm 0.81 and $85.77 \pm 1.26 \%$ ), as shown in Table 1. ANOVA analysis indicates that factor A (SPC molar concentration) had a positive significant effect, while factor B (SDC amount) and factor $\mathrm{C}$ (limonene percentage) had a negative significant effect. The effects of all factors are graphically clarified as cube plots, Figure 1A.

Considering factor (A), increasing the molar ratio of SPC would increase the lipophilicity of the vesicular system, boosting the entrapped drug (\%EE). ${ }^{8}$ Regarding factor (B), the increase in concentration of bile salts would lead to formation of micelles within the dispersion medium which lead to improved drug solubility in dispersion medium, thus lowering the $\% \mathrm{EE}{ }^{50,51}$ In addition, bile salts at high concentration have a fluidizing effect on the lipid bilayers of the vesicles, which results in loss of the entrapped drug. ${ }^{17,52}$ The effect of factor (C) could be explained as permeation enhancer that would raise the solubility of the drug and the fluidity of lipid bilayers which would lead to leakage of entrapped drug. ${ }^{53,54}$

\section{Effect of Preparation Variables on PS}

Decreasing PS is vital for permeation through skin. ${ }^{55}$ PS ranged from ( $155.5 \pm 6.65$ to $230.2 \pm 2.69 \mathrm{~nm})$, results are presented in Table 1. ANOVA analysis showed that both factor A (SPC molar concentration) and factor B (SDC amount) had positive significant effect, however, factor $\mathrm{C}$ (limonene percentage) had no significant effect. The influences of factor A (SPC molar concentration), B (SDC amount) are shown by interaction plot, Figure 1B.

Factor A could be explained on the basis that SPC has a bulk structure resulting in increasing the PS. In addition, there is a straight connection between \%EE and PS since raising PS would increase the space allowed for the lipophilic drug to be encapsulated. ${ }^{56}$ Therefore, increasing the amount SPC (factor A) would increase the lipophilicity of the medium consequently, increase both \%EE and PS. Considering factor B (SDC), bile salts have a negative charge. Therefore, increasing the concentration of bile salts would lead to formation of vesicles with large negative ZP values. Consequently, repulsion between the bilayers occurred leading to increase in PS. ${ }^{34}$ In addition, bulkiness effect of SDC due to its steroid-like structure would lead to an increase in PS. ${ }^{54}$

\section{Effect of Preparation Variables on PDI}

PDI indicates the similarity of the mixture as it becomes close to $0 .{ }^{8,57}$ PDI of all prepared formulae varied from $(0.20 \pm 0.01$ to $0.43 \pm 0.01)$, results are presented in Table 1 . These results ensure the suitable dispersion homogeneity. However ANOVA factorial results showed that no factor had significant effect on PDI.

\section{Effect of Preparation Variables on ZP}

$\mathrm{ZP}$ indicated the stability of preparation in terms of electric repulsion. Usually, system with $\mathrm{ZP}$ around $\pm 30 \mathrm{mV}$ is considered stable. ${ }^{58} \mathrm{ZP}$ of values from $(-21.2 \pm 3.25$ to -40.4 \pm 2.40 ) explained that all formulae had enough charge to prevent aggregation of particles. ANOVA analysis showed that factor A (SPC molar concentration) had a negative significant effect, while factor B (SDC amount) had a positive significant effect. Factor C (limonene percentage) had no 

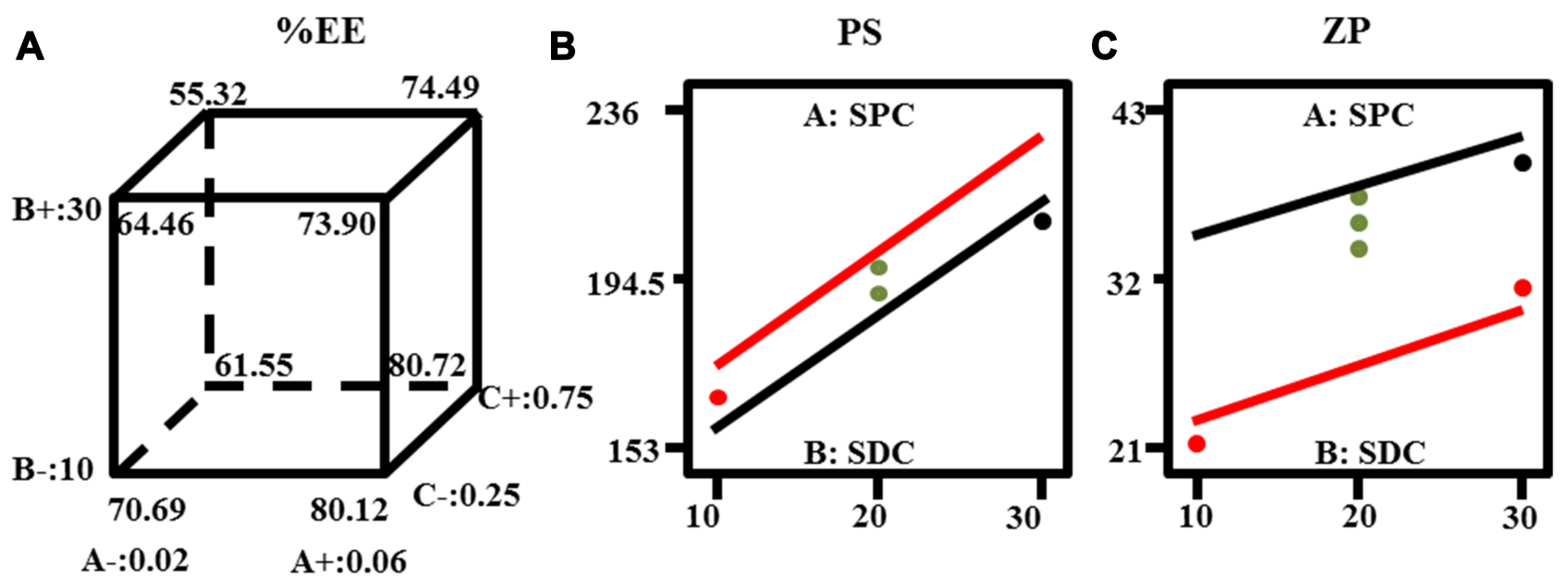
A-:0.02
$\mathrm{A}+\mathbf{0 . 0 6}$
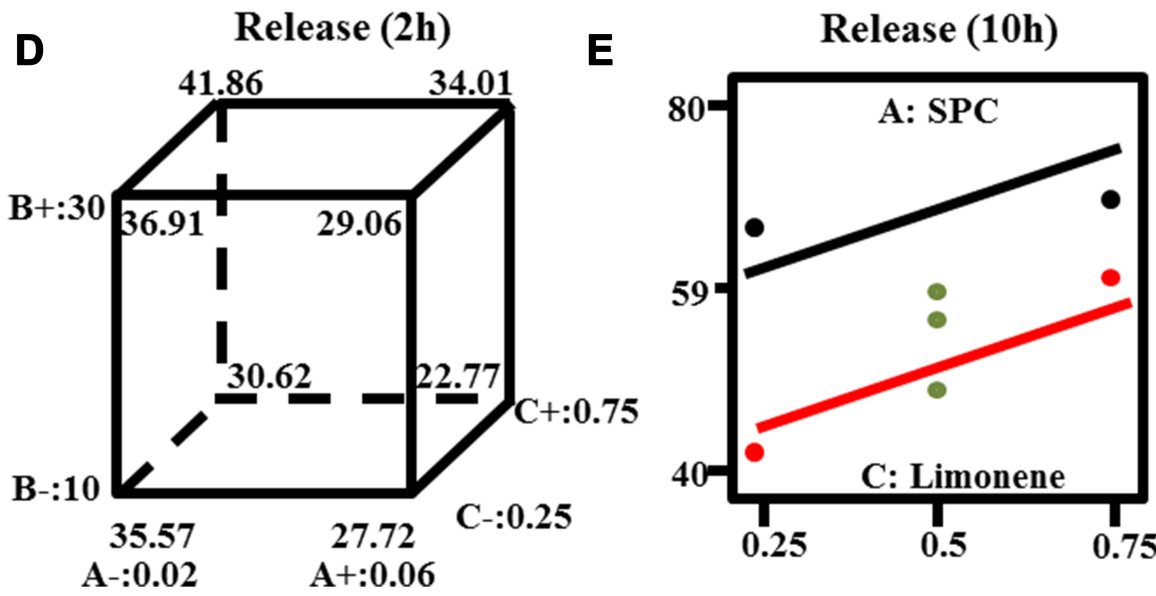

Figure I Response-plots for the effect of factor A: soy phosphatidyl choline (SPC) molar concentration (M), B: Sodium deoxycholate (SDC) amount (mg), C: limonene percentage (\%) on (A) \%EE, (B) PS and (C) ZP (D) percent drug released (two hours) and (E) percent drug released (ten hour).

significant effect. The influence of factor A (SPC molar concentration) and B (SDC amount) are illustrated by interaction plot, Figure 1C.

Factor A could be explained on the basis that SPC is neutral in nature, ${ }^{59}$ while SDC is anionic. ${ }^{54}$ In other words, increasing the molar ratio of SPC would decrease the net charge of the bilosomal system. Considering factor B, it was noticed that the absolute ZP value enlarges upon raising the bile salts concentration due to its anionic nature. ${ }^{54,60}$

Effect of Preparation Variables on in vitro Release (Percent Drug Released at 2 and $10 \mathrm{~h}$ )

In order to distinguish between different formulae, in vitro release profile for all preparation was done. Figure 2 shows complete LX release profiles. All release profiles were compared to LX suspension. Release from bilosomes formulae was significantly faster $(p<0.05)$ than LX suspension.
ANOVA analysis indicates that factor A (SPC molar concentration) had a negative significant effect at 2 and $10 \mathrm{~h}$, while factor $\mathrm{C}$ (limonene percentage) had a positive significant effect at 2 and $10 \mathrm{~h}$ Factor $\mathrm{B}$ had a positive significant effect at two hours only. The effects of these factors are revealed in Figure 1D and Figure 1E.

The effect of factor A might be explained in terms of lipophilicity. The \%EE determines the capability of the vesicle to retain the drug. The more the drug is retained inside the system, the slower the release. ${ }^{8}$ Since LX is highly lipophilic, it would prefer to remain within lipophilic system than release medium. The effect of factor $(B, C)$ could be explained through enhancement of fluidity of bilayer by bile salt and penetration enhancer effect. ${ }^{17,52,53}$

It is important to explain that bilosomal systems show biphasic release profile with initial burst followed by sustained release. Desorption of LX from the surface of bilosomal system is responsible for initial burst phase. 

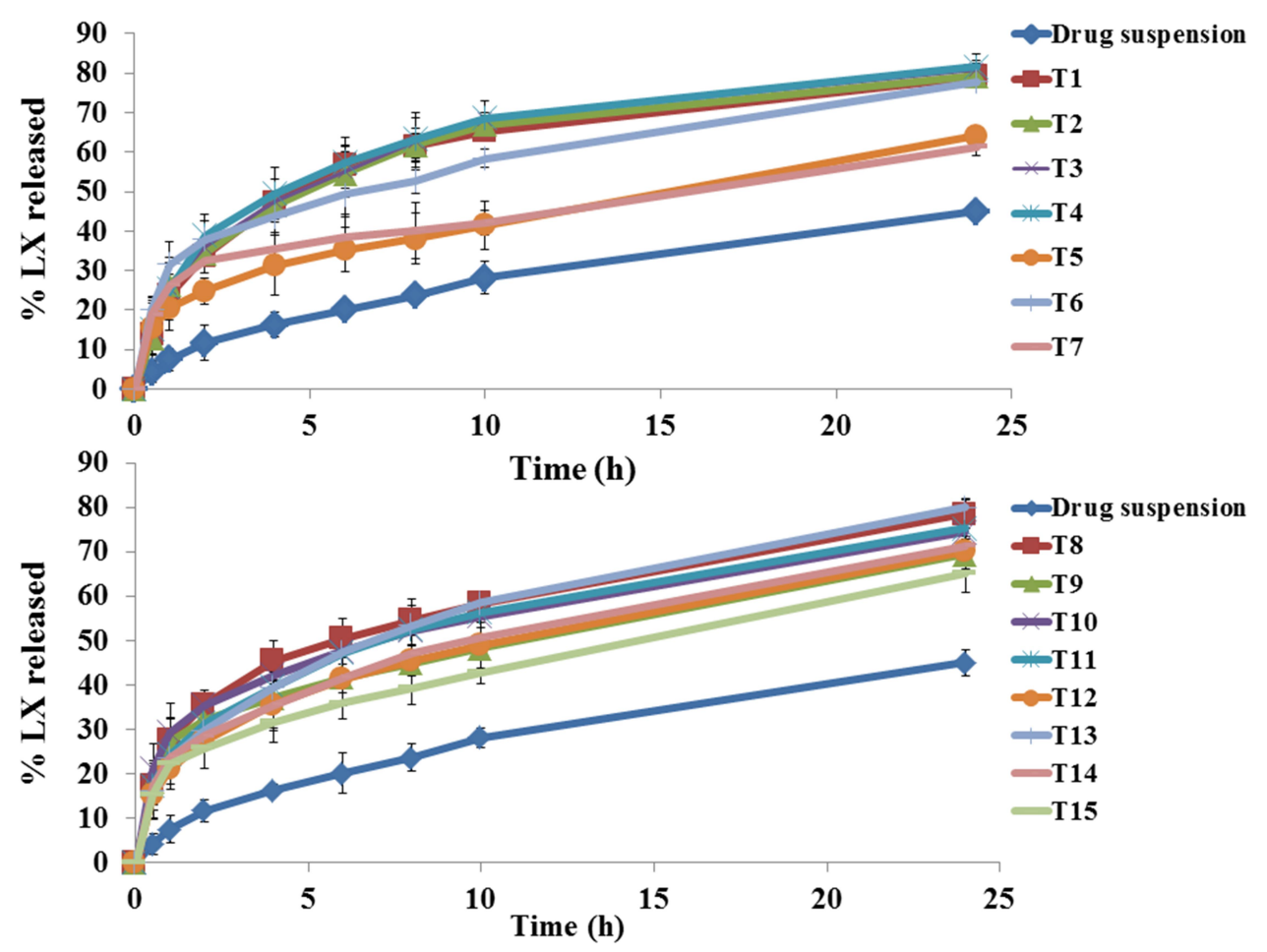

Figure 2 In vitro lornoxicam $(\mathrm{LX})$ release profiles from investigated bilosomes formulae and the $\mathrm{LX}$ suspension in phosphate buffer $(\mathrm{pH}=6.8)$ at $37 \pm 0.5^{\circ} \mathrm{C}$, mean $\pm \mathrm{SD}, \mathrm{n}=3$.

Sustained phase was due to high affinity of LX (lipophilic drug) to the bilosomal system. The Higuchi diffusion model is the most suitable model for tested formulae (highest r-square). ${ }^{28}$

\section{Validation of Optimization Process}

The composition of the optimized bilosomal formula as obtained by numerical optimization was (SPC molar concentration $=0.02 \mathrm{M}, \mathrm{SDC}$ amount $=10 \mathrm{mg}$ and limonene percentage $=0.47 \%$ with desirability $=0.736$ ). To ensure practical validity, the results of the observed and predicated responses in terms of \%EE, PS, ZP, percent drug released $(2,10 \mathrm{~h})$ were compared, as shown in Table 4. The small percent deviation as an absolute value between observed and predicated results proves the validation of our models.

\section{TEM Microscopy}

TEM is an important method to prove the results of Malvern particle size analyzer. ${ }^{61}$ Three bilosomes were measured (168.84 \pm 2.30$)$. A photomicrograph of optimized bilosomal formula is presented in Figure 3. Results showed that vesicles are spherical, homogenous with no agglomeration.

\section{Differential Scanning Calorimetry (DSC)}

DSC thermogram of LX, SDC, limonene, SPC and lyophilized optimized bilosomal formula are shown in Figure 4. DSC thermogram of LX displayed the characteristic exothermic peak at $232^{\circ} \mathrm{C}$. SDC thermogram showed an exothermic peak at $214^{\circ} \mathrm{C} .^{62}$ Limonene thermogram revealed two endothermic peaks at $72.72^{\circ} \mathrm{C}$ and $188.2^{\circ} \mathrm{C}$. SPC thermogram revealed an endothermic peak at $165^{\circ} \mathrm{C}$. The disappearance of LX peak ensures total entrapment of LX within the bilosomal

Table 4 Observed, Predicated and Percent Deviation of Optimized Bilosomal Formula

\begin{tabular}{|l|l|l|l|l|l|}
\hline Response & \% EE & PS & ZP & \% Release (2 h) & \% Release (10 h) \\
\hline Observed value & 69.17 & 161.20 & -33.95 & 35.80 & 65.96 \\
Predicated value & 69.47 & 156.91 & -34.41 & 36.15 & 66.43 \\
\% Deviation (absolute) & 0.44 & 2.73 & 1.34 & 0.97 & 0.72 \\
\hline
\end{tabular}

Abbreviations: \%EE, percent entrapment efficiency; PS, particle size; ZP, zeta potential. 


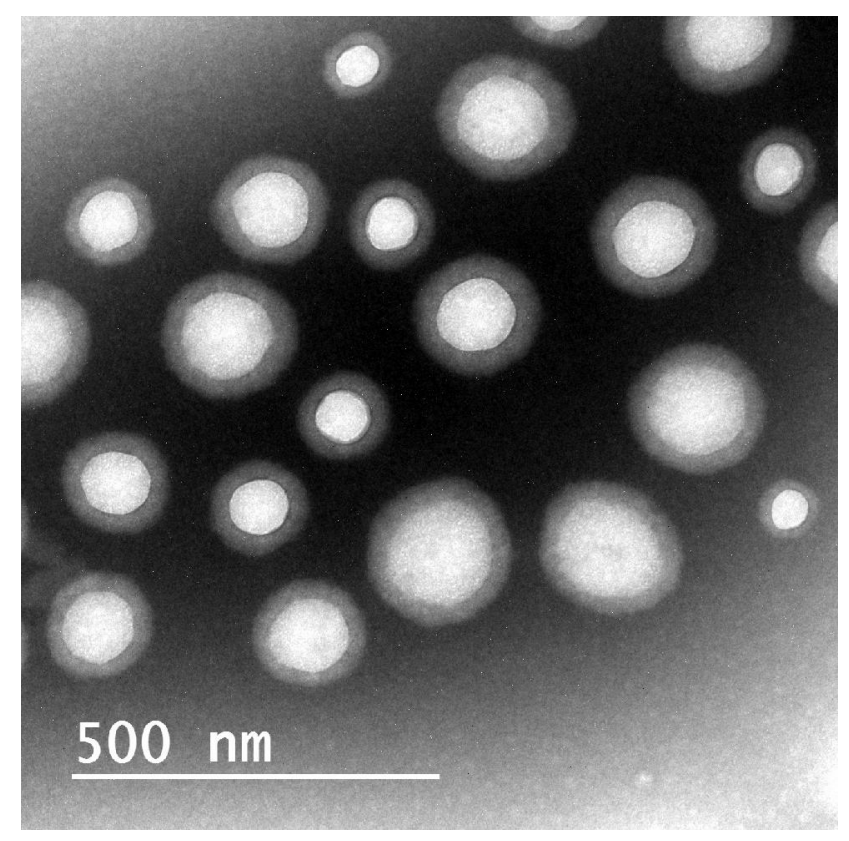

Figure 3 TEM of the optimized bilosomal formula.

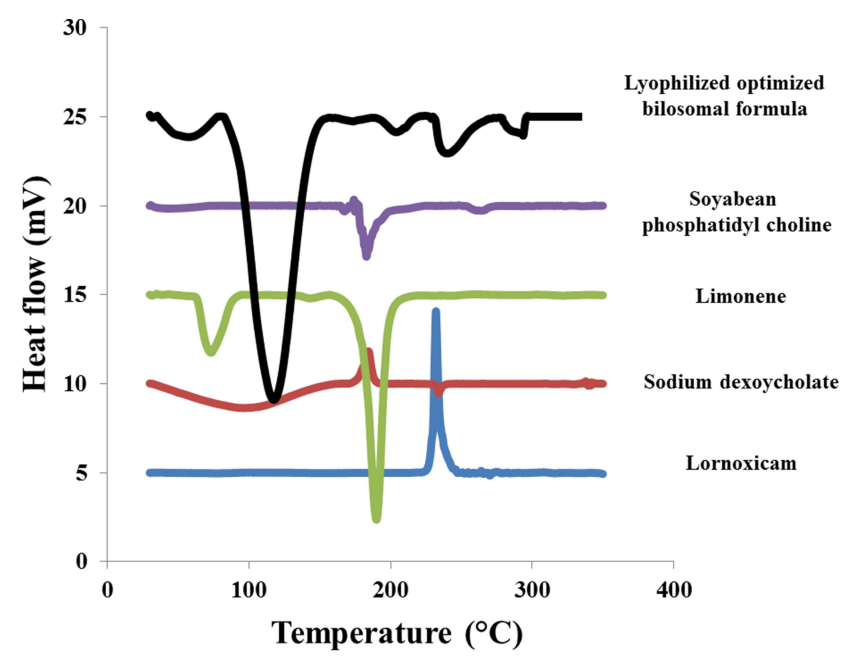

Figure 4 DSC thermogram of pure lornoxicam (LX), Sodium deoxycholate (SDC), limonene, soy phosphatidyl choline (SPC) and lyophilized optimized bilosomal formula.

system. $^{21,30}$ It also demonstrates that LX exists in its amorphous state. $^{63}$

\section{Fourier Transform Infrared Spectroscopy (FTIR)}

The FTIR spectra of LX, SDC, SPC and the lyophilized optimized bilosomal formula are shown in Figure 5. The FTIR spectra of LX are shown at 717.87 to 830.24 (C-H out-of-plane bending on the aromatic ring), 790.37 (C-Cl stretching), 1124.11 to $1187.66(\mathrm{C}-\mathrm{N}), 1158$ to 1382.94 (sulfonate group), 1596.03 and 1621.16 (bending vibrations of the amine group), 1637 (carbonyl group), and 3005.94 to $3101.29 \mathrm{~cm}^{-1}$ (aromatic ring C-H). ${ }^{9}$ The characteristic peaks SDC are shown at 2937 (aliphatic C-H), $1559 \mathrm{~cm}^{-1}$ (carbonyl group). ${ }^{62}$ The characteristic peaks SPC are shown at 1069 (-C-O-), 1465 (-C=C-), 1740 $(-\mathrm{C}=\mathrm{O}-), 2924 \mathrm{~cm}^{-1}$ (-C-H-). However, the spectrum of lyophilized optimized bilosomal formula revealed overall disappearance of LX characteristic groups which indicates the whole entrapment of the LX within the formula. Similar results were formerly noted. ${ }^{63,64}$ From the realized results of both DSC and FTIR, we can certify that LX was enclosed within the optimized bilosomal formula.

\section{Effect of Storage}

After three months of storage the optimized bilosomal formula showed clear appearance. The responses of both stored optimized bilosomal formula and freshly prepared form are shown in Table 5. No critical change at $(p>0.05)$ in $\% \mathrm{EE}$, PS, ZP and release after $(2,10 \mathrm{~h})$ of the kept samples when referred to the fresh formula by one-way ANOVA. High stability of optimized bilosomal formula is due to high negative charge due to the anionic nature of SDC, ${ }^{54}$ addition of lecithin to bile salts lead to formation of mixed micelles ${ }^{65}$ and nanosize of the optimized bilosomal formula.

\section{Ex vivo Permeation Study Through Rat Skin}

The permeation profiles of LX from optimized bilosomal gel and LX suspension gel through rat skin were revealed in Figure 6. Optimized bilosomal gel showed better amount of LX permeated after $10 \mathrm{~h}(\mathrm{Q} 10 \mathrm{~h}$ of optimized bilosomal gel $\left.=326.10 \pm 1.57 \mu \mathrm{g} / \mathrm{cm}^{2}\right)$ compared to $(\mathrm{Q} 10 \mathrm{~h}$ of LX suspension gel $\left.=39.43 \pm 1.32 \mu \mathrm{g} / \mathrm{cm}^{2}\right)$. Optimized bilosomal gel revealed greater flux $\left(\mathrm{J}_{\max }\right.$ of the optimized bilosomal gel $\left.=32.61 \pm 0.16 \mu \mathrm{g} / \mathrm{cm}^{2} / \mathrm{h}\right)$ compared to $\left(\mathrm{J}_{\max }\right.$ of LX suspension gel $=3.94 \pm 0.13 \mu \mathrm{g} / \mathrm{cm}^{2} / \mathrm{h}$ ). Q10 optimized bilosomal gel was subjected to one-way ANOVA which ensure that Q10 optimized bilosomal gel significantly higher than $\mathrm{Q} 10 \mathrm{~h}$ LX suspension gel $(p<0.05)$. The enhancement ratio of optimized bilosomal gel is (8.27). So the optimized bilosomal formula could both enhance entrapment and permeation of LX.

Bilosomes showed considerably greater drug permeation compared to LX suspension gel. It is obvious that permeation of bilosomes through the skin occurs through three phases; an initial short "burst" within an hour, 


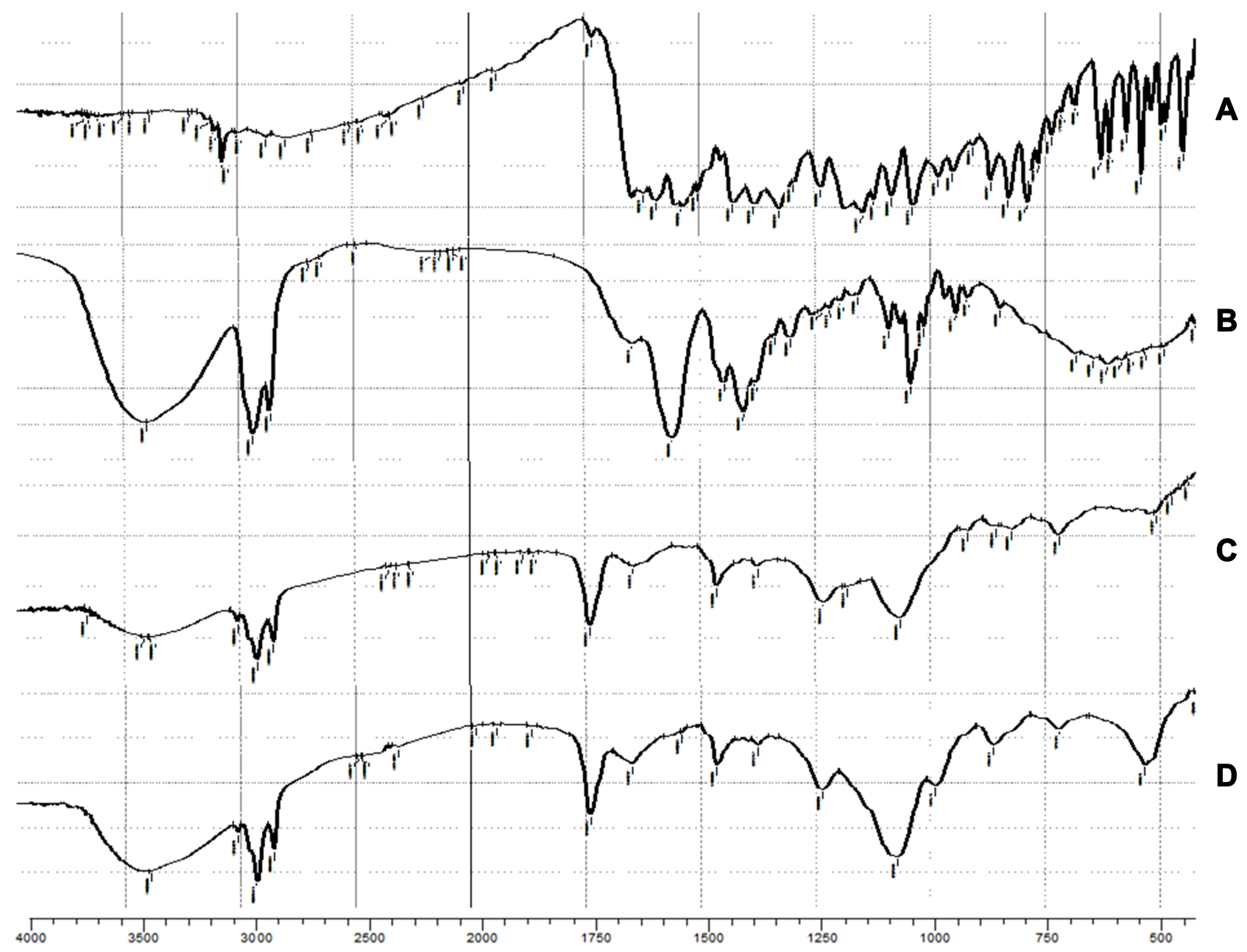

Figure 5 The FTIR spectra of (A) pure lornoxicam (LX), (B) sodium deoxycholate (SDC), (C) soy phosphatidyl choline (SPC) and (D) the lyophilized optimized bilosomal formula.

followed by slow increase up to four hours and finally saturation level that extends until the end of the study.

Bilosomes permeate through the skin via different routes; corneocytes (intracellular/transcellular route), between the corneocytes (intercellular route), and via the appendages (sweat glands or hair follicles). ${ }^{66}$ Bile salts fluidize SC lipids so improving the flexibility and offering a greater chance that allows them to permeate through intact skin. In addition, bile salts can isolate calcium ions that are responsible for the integrity of the tight junction, causing them to become more leaker. ${ }^{15}$

\section{In vivo Study}

Assessment of Anti-inflammatory Effect

Figure 7 reveals greatest value of edema in group I (negative control) with a mean increase in edema of $95.96 \%$ after six

Table 5 Stability Study for Optimized Bilosomal Formula

\begin{tabular}{|l|l|l|l|}
\hline Parameter & Fresh & After 3 Month Storage at (4-8 $\left.{ }^{\circ} \mathbf{C}\right)$ & Probability ( $\mathbf{p})$ \\
\hline \%EE & $69.17 \pm 2.50$ & $66.30 \pm 2.96$ & 0.405 \\
PS & $161.20 \pm 3.68$ & $166.70 \pm 1.13$ & 0.181 \\
ZP & $-33.95 \pm 0.49$ & $34.10 \pm 1.13$ & 0.879 \\
\% drug released (2 h) & $35.80 \pm 2.16$ & $37.24 \pm 2.11$ & 0.571 \\
\% drug released (10 h) & $65.96 \pm 0.83$ & $63.51 \pm 3.30$ & 0.416 \\
\hline
\end{tabular}

Abbreviations: \%EE, percent entrapment efficiency; PS, particle size; ZP, zeta potential. 


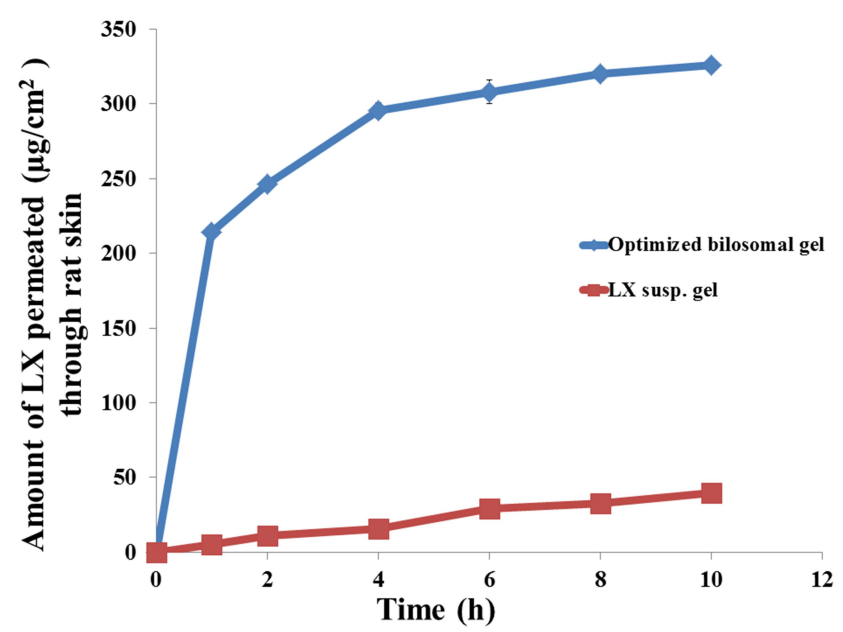

Figure 6 The ex vivo transport profiles of optimized bilosomal gel compared to that from lornoxicam suspension gel through rat skin at $37 \pm 0.5^{\circ} \mathrm{C}$, mean $\pm S D, n=3$.

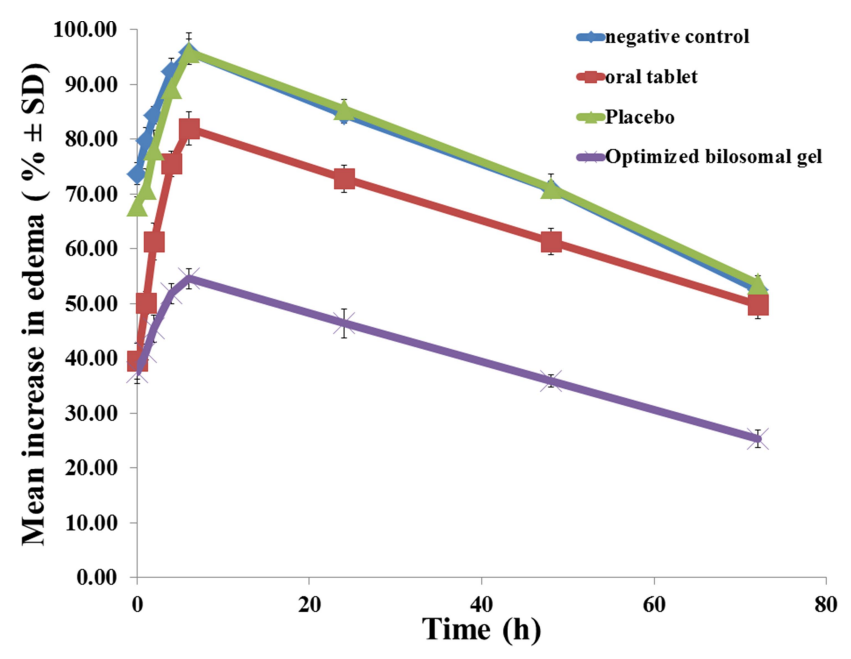

Figure 7 Effect of the optimized bilosomal gel on inhibition of edema in the hind paw of rats.

hours, followed by a sharp decrease to $52.45 \%$ after $72 \mathrm{~h}$. The size of edema in group II (oral market tablet) reached its greatest value after six hours with a mean increase in edema of $(81.94 \%)$, then a slightly sharp reduction reaching (49.75\%) after $72 \mathrm{~h}$. Rats in group III (placebo) reveals highest value after six hours (95.88\%) and then it decreased to (53.58\%) after $72 \mathrm{~h}$. Group IV (optimized bilosomal gel) revealed the highest value in edema size after six hours with a mean increase of (54.54\%), which then reduced to (25.25\%) after $72 \mathrm{~h}$. Group IV showed the least AUC indicating its superior anti-inflammatory activity compared to market product $(p<0.05)$.

\section{Assessment of Antinociceptive Activity}

Table 6 shows the number of writhes detected and the degree of inhibition realized by the optimized bilosomal gel and
Table 6 Effect of Different Formulae on Acetic Acid-induced Writhing in Mice

\begin{tabular}{|l|l|l|}
\hline Preparation & $\begin{array}{l}\text { Number of Writhes } \\
\text { (Mean } \pm \text { SE) }\end{array}$ & $\begin{array}{l}\text { Percent } \\
\text { Inhibition (\%) }\end{array}$ \\
\hline Negative control & $59.75 \pm 2.75$ & - \\
\hline $\begin{array}{l}\text { Oral market } \\
\text { product }\end{array}$ & $21.50 \pm 1.29$ & 64.02 \\
\hline $\begin{array}{l}\text { Optimized } \\
\text { bilosomal gel }\end{array}$ & $15.25 \pm 1.26$ & 74.48 \\
\hline
\end{tabular}

linked to the market product. The examined optimized bilosomal gel considerably lowered the number of writhes related to commercial product $(p<0.05)$. This matches the results of the former edema test, where the formula proved to be more effective as well.

\section{Skin Irritancy Test}

The recorded zero scores for the optimized bilosomal gel (by visual examination) indicates the absence of any dermal irritation. No clear erythema or edema was observed on rats' skin after application for two days.

\section{In vivo Histopathological Study}

Considering group I (negative control); the stained rat skin revealed no histopathological modification and the normal intact histological pattern of the epidermal and dermal layers as well as the underlying subcutaneous tissue and skeletal muscle as shown in Figure 8A. Considering group II (optimized bilosomal gel); neither inflammation nor skin irritation was revealed in the examined sections with intact connective tissue, as shown in Figure 8B. Considering group III (positive control); both edema and inflammatory cells infiltration were revealed in the examined sections with lose of the glands in the dermis, as shown in Figure 8C. As a conclusion, bilosomal system was safe and could be used topically without any irritation or inflammation consequences.

\section{In vivo Permeation}

Imaging of RhB in various skin layers by CLSM is shown in Figure 9. RhB-loaded bilosomal gel exhibited deeper penetration $(108 \mu \mathrm{m})$ than $\mathrm{RhB}$ gel $(48 \mu \mathrm{m})$. These results highlighted the efficacy of the bilosomal system in terms of in vivo permeability when matched to the reference solution gel. This matches the results of former ex vivo permeation study. 
A

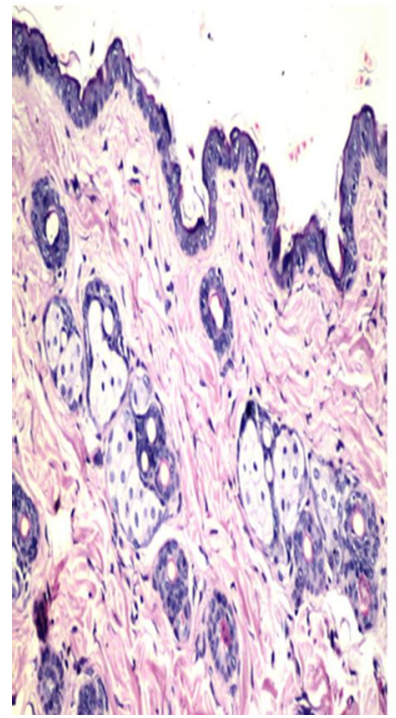

B

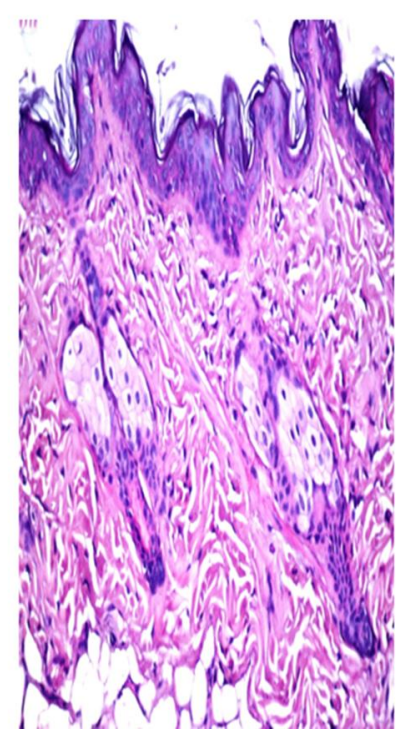

C

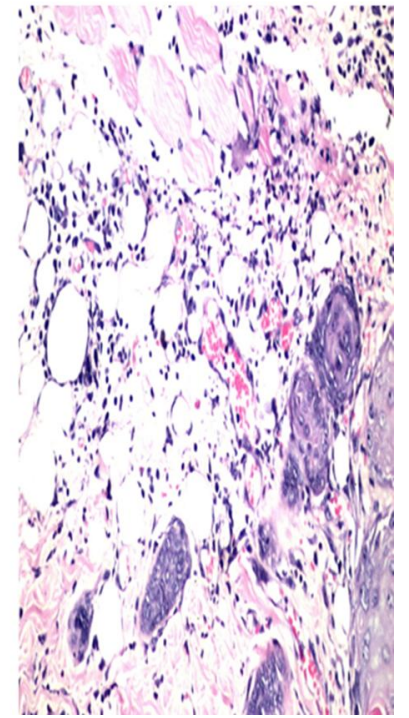

Figure 8 Photomicrographs showing histopathological sections (H\&E stained) of (A) rat skin treated with normal saline (B) rat skin treated with optimized bilosomal gel and (C) rat skin treated with isopropanol (positive control).
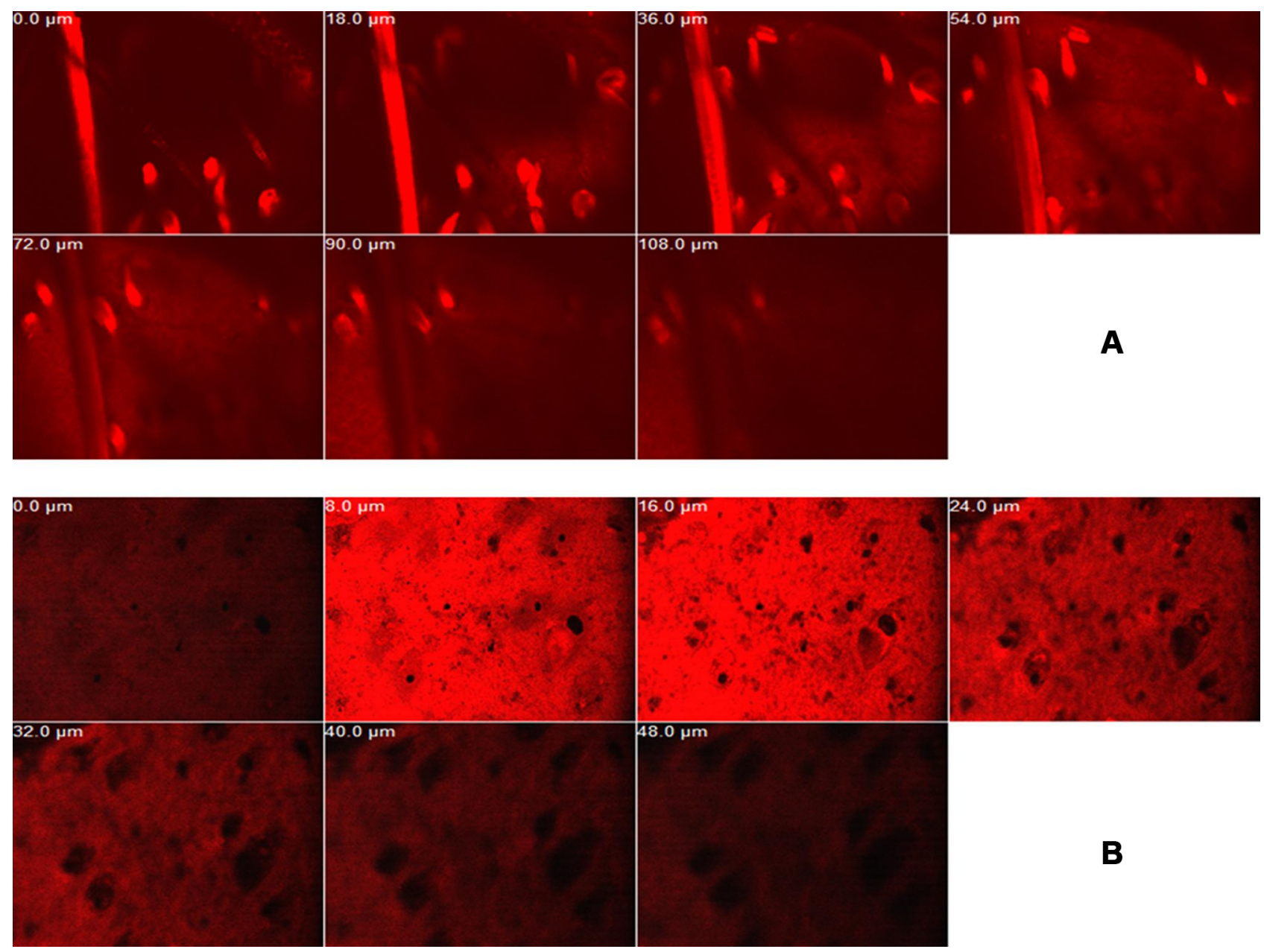

Figure 9 Confocal laser scanning micrographs of RhB bilosomal gel (A) and RhB solution gel (B). 


\section{Conclusion}

In this study, bilosomes were successfully formulated using thin film hydration technique. The optimized bilosomal formula established small particle size (161.20 \pm 3.68$)$, high percent entrapment efficiency $(69.17 \% \pm 2.50)$ and high physicochemical stability. The presence of LX within the structure of bilosomes was revealed by DSC and FTIR studies. Spherical vesicles with no aggregation were proved by TEM. It has been clarified that LX-loaded bilosomes had succeeded to enhance the ex vivo skin permeation (326.10 $\pm 1.57 \mu \mathrm{g} / \mathrm{cm}^{2}$ ). Safety of optimized bilosomal gel was demonstrated by in vivo skin irritancy and histopathological studies. Anti-inflammatory and analgesic activities of the optimized bilosomal gel were proved through in vivo studies. CLSM proved the superior in vivo permeation of bilosomal system. Overall, previous results indicate that inclusion of LX within bilosomes system is a promising technique to enhance transdermal delivery of the drug.

\section{Funding}

This research did not receive any specific grant from funding agencies in the public, commercial, or not-forprofit sectors.

\section{Disclosure}

The authors report no conflicts of interest in this work and declare that they have no known competing financial interests or personal relationships that could have appeared to influence the work reported in this paper.

\section{References}

1. Du J, Li X, Zhao H, et al. Nanosuspensions of poorly water-soluble drugs prepared by bottom-up technologies. Int J Pharm. 2015;495 (2):738-749. doi:10.1016/j.ijpharm.2015.09.021.

2. Yadav SK, Mishra S, Mishra B. Eudragit-Based Nanosuspension of Poorly Water-Soluble Drug: formulation and In Vitro-In Vivo Evaluation. AAPS PharmSciTech. 2012;13(4):1031-1044. doi:10.1208/s12249-012-9833-0.

3. Gao L, Liu G, Ma J, Wang X, Zhou L, Li X. Drug nanocrystals: in vivo performances. $J$ Control Release. 2012;160(3):418-430. doi:10.1016/j.jconrel.2012.03.013.

4. Lorenz IH, Egger K, Schubert H, et al. Lornoxicam characteristically modulates cerebral pain-processing in human volunteers: a functional magnetic resonance imaging study. Br J Anaesth. 2008;100(6):827833. doi:10.1093/bja/aen082.

5. Atzpodien E, Mehdi N, Clarke D, Radhofer-Welte S. Subacute and chronic oral toxicity of lornoxicam in cynomolgus monkeys. Food Chem Toxicol. 1997;35(5):465-474. doi:10.1016/s0278-6915(97) 00019-7.

6. Prajapati P, Vaghela V, Rawtani D, Patel H, Kubavat J, Baraiya D. Azeotropic mixture used for development and validation of Lornoxicam in bulk and its tablet dosage form by spectrophotometric method. J Pharm Analysis. 2012;2(4):306-309. doi:10.1016/j.jpha.2012.02.004.
7. Tawfeek HM, Saleem IY, Roberts M. Dissolution enhancement and formulation of rapid-release lornoxicam mini-tablets. J Pharm Sci. 2014;103(8):2470-2483. doi:10.1002/jps.24073.

8. Abdelbary GA, Aburahma MH. Oro-dental mucoadhesive proniosomal gel formulation loaded with lornoxicam for management of dental pain. J Liposome Res. 2015;25(2):107-121. doi:10.3109/ 08982104.2014.941861.

9. Joseph J, B.N. VH, D. RD. Experimental optimization of Lornoxicam liposomes for sustained topical delivery. Eur $j$ Pharm Sci. 2018;112:38-51. doi:10.1016/j.ejps.2017.10.032.

10. Shah H, Nair AB, Shah J, Bharadia P, Al-Dhubiab BE. Proniosomal gel for transdermal delivery of lornoxicam: optimization using factorial design and in vivo evaluation in rats. DARU Journal of Pharmaceutical Sciences. 2019;27(1):59-70. doi:10.1007/s40199019-00242-x.

11. Kumbhar D, Wavikar P, Vavia P. Niosomal gel of lornoxicam for topical delivery: in vitro assessment and pharmacodynamic activity. AAPS PharmSciTech. 2013;14(3):1072-1082. doi:10.1208/s12249013-9986-5.

12. Jain S, Jain N, Bhadra D, Tiwary AK, Jain NK. Transdermal delivery of an analgesic agent using elastic liposomes: preparation, characterization and performance evaluation. <![CDATA[Current Drug Delivery]]>. 2005;2(3):223-233. doi:10.2174/1567201054368020.

13. Bragagni M, Mennini N, Maestrelli F, Cirri M, Mura P. Comparative study of liposomes, transfersomes and ethosomes as carriers for improving topical delivery of celecoxib. Drug Deliv. 2012;19 (7):354-361. doi:10.3109/10717544.2012.724472.

14. Duangjit S, Opanasopit P, Rojanarata T, Ngawhirunpat T. Evaluation of meloxicam-loaded cationic transfersomes as transdermal drug delivery carriers. AAPS PharmSciTech. 2013;14(1):133-140. doi:10.1208/s12249-012-9904-2.

15. Al-Mahallawi AM, Abdelbary AA, Aburahma MH. Investigating the potential of employing bilosomes as a novel vesicular carrier for transdermal delivery of tenoxicam. Int $J$ Pharm. 2015;485(12):329-340. doi:10.1016/j.ijpharm.2015.03.033.

16. Conacher M, Alexander J, Brewer JM. Oral immunisation with peptide and protein antigens by formulation in lipid vesicles incorporating bile salts (bilosomes). Vaccine. 2001;19(20-22):2965-2974. doi:10.1016/s0264-410x(00)00537-5.

17. Aburahma MH. Bile salts-containing vesicles: promising pharmaceutical carriers for oral delivery of poorly water-soluble drugs and peptide/protein-based therapeutics or vaccines. Drug Deliv. 2016;23 (6): 1847-1867. doi:10.3109/10717544.2014.976892.

18. Jain S, Harde H, Indulkar A, Agrawal AK. Improved stability and immunological potential of tetanus toxoid containing surface engineered bilosomes following oral administration. Nanomedicine: Nanotechnology, Biology and Medicine. 2014;10(2):431-440. doi:10.1016/j.nano.2013.08.012.

19. Wilkhu JS, McNeil SE, Anderson DE, Perrie Y. Characterization and optimization of bilosomes for oral vaccine delivery. $J$ Drug Target. 2013;21(3):291-299. doi:10.3109/1061186X.2012.747528.

20. Huang Y-B, Tsai Y-H, Yang W-C, Chang J-S, Wu P-C, Takayama $\mathrm{K}$. Once-daily propranolol extended-release tablet dosage form: formulation design and in vitro/in vivo investigation. Eur $j$ Pharm Biopharm. 2004;58(3):607-614. doi:10.1016/j. ejpb.2004.03.037.

21. Habib BA, Sayed S, Elsayed GM. Enhanced transdermal delivery of ondansetron using nanovesicular systems: fabrication, characterization, optimization and ex-vivo permeation study-Box-Cox transformation practical example. Eur j Pharm Sci. 2018;115:352-361. doi:10.1016/j.ejps.2018.01.044.

22. Abdelbary AA, AbouGhaly MHH. Design and optimization of topical methotrexate loaded niosomes for enhanced management of psoriasis: application of Box-Behnken design, in-vitro evaluation and in-vivo skin deposition study. Int J Pharm. 2015;485(1-2):235243. doi:10.1016/j.ijpharm.2015.03.020. 
23. Elsayed I, Sayed S. Tailored nanostructured platforms for boosting transcorneal permeation: box-Behnken statistical optimization, comprehensive in vitro, ex vivo and in vivo characterization. <! [CDATA [International Journal of Nanomedicine]]>. 2017;12:7947-7962. doi:10.2147/IJN.S150366.

24. Scognamiglio I, De Stefano D, Campani V, et al. Nanocarriers for topical administration of resveratrol: a comparative study. Int $J$ Pharm. 2013;440(2):179-187. doi:10.1016/j.ijpharm.2012.08.009.

25. Das S, Ng WK, Kanaujia P, Kim S, Tan RBH. Formulation design, preparation and physicochemical characterizations of solid lipid nanoparticles containing a hydrophobic drug: effects of process variables. Colloids and Surfaces B: Biointerfaces. 2011;88(1):483-489. doi:10.1016/j.colsurfb.2011.07.036.

26. Yang -Z-Z, Zhang Y-Q, Wang -Z-Z, Wu K, Lou J-N, Qi X-R. Enhanced brain distribution and pharmacodynamics of rivastigmine by liposomes following intranasal administration. Int J Pharm. 2013;452(1-2):344-354. doi:10.1016/j.ijpharm.2013.05.009.

27. Higuchi T. Mechanism of Sustained-Action Medication. Theoretical Analysis of Rate of Release of Solid Drugs Dispersed in Solid Matrices. J Pharm Sci. 1963;52(12):1145-1149. doi:10.1002/jps.2600521210.

28. Gaber NN, Darwis Y, Peh -K-K, Tan YT-F. Characterization of polymeric micelles for pulmonary delivery of beclomethasone dipropionate. J Nanosci Nanotechnol. 2006;6(9):3095-3101. doi:10.1166/ jnn.2006.426.

29. Sayed S, Habib BA, Elsayed GM. Tri-block co-polymer nanocarriers for enhancement of oral delivery of felodipine: preparation, in vitro characterization and ex vivo permeation. J Liposome Res. 2018;28 (3):182-192. doi:10.1080/08982104.2017.1327541.

30. Shamma RN, Sayed S, Sabry NA, El-Samanoudy SI. Enhanced skin targeting of retinoic acid spanlastics: in vitro characterization and clinical evaluation in acne patients. J Liposome Res. 2019;29(3):283290. doi:10.1080/08982104.2018.1552706.

31. Al-Mahallawi AM, Khowessah OM, Shoukri RA. Enhanced non invasive trans -tympanic delivery of ciprofloxacin through encapsulation into nano-spanlastic vesicles: fabrication, in-vitro characterization, and comparative ex-vivo permeation studies. Int J Pharm. 2017;522(1-2):157-164. doi:10.1016/j.ijpharm.2017.03.005.

32. Gaikwad PJ, Sutar SV, Kulkarni AS, Mane MB, Patil SS. RP-HPLC method for degradation study of Lornoxicam. Der Pharm Lett. 2016;8(3):62-71.

33. AbdelSamie SM, Kamel AO, Sammour OA, Ibrahim SM. Terbinafine hydrochloride nanovesicular gel: in vitro characterization, ex vivo permeation and clinical investigation. Eur j Pharm Sci. 2016;88:91100. doi:10.1016/j.ejps.2016.04.004.

34. El Zaafarany GM, Awad GAS, Holayel SM, Mortada ND. Role of edge activators and surface charge in developing ultradeformable vesicles with enhanced skin delivery. Int J Pharm. 2010;397(12):164-172. doi:10.1016/j.ijpharm.2010.06.034.

35. Sayed S, Elsayed I, Ismail MM. Optimization of $\beta$-cyclodextrin consolidated micellar dispersion for promoting the transcorneal permeation of a practically insoluble drug. Int J Pharm. 2018;549(12):249-260. doi:10.1016/j.ijpharm.2018.08.001.

36. Abd-Elsalam WH, El-Zahaby SA, Al-Mahallawi AM. Formulation and in vivo assessment of terconazole-loaded polymeric mixed micelles enriched with Cremophor EL as dual functioning mediator for augmenting physical stability and skin delivery. Drug Deliv. 2018;25(1):484-492. doi:10.1080/10717544.2018.1436098.

37. Ammar HO, Ghorab M, El-Nahhas SA, Higazy IM. Proniosomes as a carrier system for transdermal delivery of tenoxicam. Int J Pharm. 2011;405(1-2):142-152. doi:10.1016/j.ijpharm.2010.11.003.

38. Selye H. Participation of Adrenal Cortex in Pathogenesis of Arthritis. BMJ. 1949;2(4637):1129-1135. doi:10.1136/bmj.2.4637.1129

39. Moura AC, Silva EL, Fraga MC, Wanderley AG, Afiatpour P, Maia MB. Antiinflammatory and chronic toxicity study of the leaves of Ageratum conyzoides L. in rats. Phytomedicine. 2005;12(1-2):138142. doi:10.1016/j.phymed.2003.12.003.
40. Adzu B, Amos S, Wambebe C, Gamaniel K. Antinociceptive activity of Zizyphus spina-christi root bark extract. Fitoterapia. 2001;72 (4):344-350. doi:10.1016/s0367-326x(00)00289-6.

41. Young H-Y, Luo Y-L, Cheng H-Y, Hsieh W-C, Liao J-C, Peng W-H. Analgesic and anti-inflammatory activities of [6]-gingerol. $J$ Ethnopharmacol. 2005;96(1-2):207-210. doi:10.1016/j.jep.2004.09.009.

42. Mandawgade SD, Patravale VB. Development of SLNs from natural lipids: application to topical delivery of tretinoin. Int J Pharm. 2008;363(1-2):132-138. doi:10.1016/j.ijpharm.2008.06.028.

43. Bancroft D, Steven A, Turner R. Theory and Practice of Histological Techniques, 6th ed. Churchill Livingstone. 2007;14.

44. Yu W, Jiang G, Zhang Y, Liu D, Xu B, Zhou J. Polymer microneedles fabricated from alginate and hyaluronate for transdermal delivery of insulin. Materials Science and Engineering: C. 2017;80:187-196. doi:10.1016/j.msec.2017.05.143.

45. Cevc G, Schatzlein A, Richardsen H. Ultradeformable lipid vesicles can penetrate the skin and other semi-permeable barriers unfragmented. Evidence from double label CLSM experiments and direct size measurements. Biochimica Et Biophysica Acta (BBA) - Biomembranes. 2002;1564(1):21-30. doi:10.1016/s0005-2736(02)00401-7.

46. Shamma RN, Aburahma MH. Follicular delivery of spironolactone via nanostructured lipid carriers for management of alopecia. Int $J$ Nanomedicine. 2014;9:5449-5460. doi:10.2147/IJN.S73010.

47. Hathout RM, Nasr M. Transdermal delivery of betahistine hydrochloride using microemulsions: physical characterization, biophysical assessment, confocal imaging and permeation studies. Colloids and Surfaces B: Biointerfaces. 2013;110:254-260. doi:10.1016/j. colsurfb.2013.05.007.

48. Araujo J, Gonzalez-Mira E, Egea MA, Garcia ML, Souto EB. Optimization and physicochemical characterization of a triamcinolone acetonide-loaded NLC for ocular antiangiogenic applications. Int $J$ Pharm. 2010;393(1-2):167-175. doi:10.1016/j.ijpharm.2010.03.034.

49. Zhang Y, Wang X, Lin X, Liu X, Tian B, Tang X. High azithromycin loading powders for inhalation and their in vivo evaluation in rats. Int J Pharm. 2010;395(1-2):205-214. doi:10.1016/j.ijpharm.2010.05.043.

50. Mahmood S, Taher M, Mandal UK. Experimental design and optimization of raloxifene hydrochloride loaded nanotransfersomes for transdermal application. Int J Nanomedicine. 2014;9:4331-4346. doi:10.2147/IJN.S65408.

51. Niu M, Tan Y, Guan P, et al. Enhanced oral absorption of insulinloaded liposomes containing bile salts: a mechanistic study. Int $J$ Pharm. 2014;460(1-2):119-130. doi:10.1016/j.ijpharm.2013.11.028.

52. Niu M, Lu Y, Hovgaard L, Wu W. Liposomes containing glycocholate as potential oral insulin delivery systems: preparation, in vitro characterization, and improved protection against enzymatic degradation. Int J Nanomedicine. 2011;6:1155-1166. doi:10.2147/IJN.S19917.

53. Salama HA, Mahmoud AA, Kamel AO, Abdel Hady M, Awad GAS. Brain delivery of olanzapine by intranasal administration of transfersomal vesicles. J Liposome Res. 2012;22(4):336-345. doi:10.3109/ 08982104.2012.700460.

54. Abdelbary AA, Abd-Elsalam WH, Al-Mahallawi AM. Fabrication of novel ultradeformable bilosomes for enhanced ocular delivery of terconazole: in vitro characterization, ex vivo permeation and in vivo safety assessment. Int $J$ Pharm. 2016;513(1-2):688-696. doi:10.1016/j.ijpharm.2016.10.006.

55. Verma DD, Verma S, Blume G, Fahr A. Particle size of liposomes influences dermal delivery of substances into skin. Int J Pharm. 2003;258(1-2):141-151. doi:10.1016/s0378-5173(03)00183-2.

56. Hathout RM, Mansour S, Mortada ND, Guinedi AS. Liposomes as an ocular delivery system for acetazolamide: in vitro and in vivo studies. AAPS PharmSciTech. 2007;8(1):1. doi:10.1208/pt0801001.

57. Younes NF, Abdel-Halim SA, Elassasy AI. Solutol HS15 based binary mixed micelles with penetration enhancers for augmented corneal delivery of sertaconazole nitrate: optimization, in vitro, ex vivo and in vivo characterization. Drug Deliv. 2018;25(1):17061717. doi:10.1080/10717544.2018.1497107. 
58. Muller RH, Jacobs C, Kayser O. Nanosuspensions as particulate drug formulations in therapy. Rationale for development and what we can expect for the future. Adv Drug Deliv Rev. 2001;47(1):3-19. doi:10.1016/s0169-409x(00)00118-6.

59. Schlieper P, Medda PK, Kaufmann R. Drug-induced zeta potential changes in liposomes studied by laser Doppler spectroscopy. Biochimica Et Biophysica Acta (BBA) - Biomembranes. 1981;644 (2):273-283. doi:10.1016/0005-2736(81)90385-0.

60. Basha M, Abd El-Alim SH, Shamma RN, Awad GEA. Design and optimization of surfactant-based nanovesicles for ocular delivery of Clotrimazole. J Liposome Res. 2013;23(3):203-210. doi:10.3109/ 08982104.2013.788025.

61. Lim WM, Rajinikanth PS, Mallikarjun C, Kang YB. Formulation and delivery of itraconazole to the brain using a nanolipid carrier system. Int J Nanomedicine. 2014;9:2117-2126. doi:10.2147/IJN.S57565.

62. Suzuki H, Ogawa M, Hironaka K, Ito K, Sunada H. A nifedipine coground mixture with sodium deoxycholate. I. Colloidal particle formation and solid-state analysis. Drug Dev Ind Pharm. 2001;27 (9):943-949. doi:10.1081/ddc-100107675.
63. Song Y, Tian Q, Huang Z, et al. Self-assembled micelles of novel amphiphilic copolymer cholesterol-coupled F68 containing cabazitaxel as a drug delivery system. Int J Nanomedicine. 2014;9:23072317. doi:10.2147/IJN.S61220.

64. Abdelbary G, Makhlouf A. Adoption of polymeric micelles to enhance the oral bioavailability of dexibuprofen: formulation, invitro evaluation and in-vivo pharmacokinetic study in healthy human volunteers. Pharm Dev Technol. 2014;19(6):717-727. doi:10.3109/10837450.2013.823994.

65. Tan Y, Qi J, Lu Y, Hu F, Yin Z, Wu W. Lecithin in mixed micelles attenuates the cytotoxicity of bile salts in Caco-2 cells. Toxicol Vitro. 2013;27(2):714-720. doi:10.1016/j.tiv.2012.11.018.

66. Honeywell-Nguyen PL, Bouwstra JA. Vesicles as a tool for transdermal and dermal delivery. Drug Discovery Today: Technologies. 2005;2(1):67-74. doi:10.1016/j.ddtec.2005.05.003.

\section{Publish your work in this journal}

The International Journal of Nanomedicine is an international, peerreviewed journal focusing on the application of nanotechnology in diagnostics, therapeutics, and drug delivery systems throughout the biomedical field. This journal is indexed on PubMed Central, MedLine, CAS, SciSearch ${ }^{\mathbb{R}}$, Current Contents ${ }^{\mathbb{R}} /$ Clinical Medicine, $^{-}$
Journal Citation Reports/Science Edition, EMBase, Scopus and the Elsevier Bibliographic databases. The manuscript management system is completely online and includes a very quick and fair peer-review system, which is all easy to use. Visit http://www.dovepress.com/ testimonials.php to read real quotes from published authors. 